\title{
Mental Fatigue Measurement using Visual Synchronization Tasks
}

\author{
Kyota Aoki \\ Department of Information Systems Science, Graduate School of Engineering, Utsunomiya University \\ 7-1-2, Yoto, Utsunomiya, JAPAN \\ $+81-28-689-6247$ \\ kyota@is.utsunomiya-u.ac.jp
}

\begin{abstract}
There are peg-board test and tap synchronization test as a method to measure the motion control function. These tests measure only the result of motion, and do not the process. By measuring the whole process from the beginning to the end of a test, it is possible to obtain data that are more detailed. The through measurement of a motion process enables to measure the precise performance of the motor control function. This paper discusses visual synchronization task to measure the performance of our brain using periodic motion of both hands for estimating the mental fatigue caused from a mental workload. A visual synchronization task requests a subject to flip the palms of both hands synchronizing the displayed hands' motion. The method measures the poses of both perms through the task. From the measurements in the process of this task, the method evaluates the performance of the motion control function of the subject. This paper discusses the descriptive power of the visual synchronization task to estimate the mental fatigue. The visual synchronization task is safe, because there is no need to attach the device to a subject nor to make gross motions. The task is short to complete. This paper presents the experiments and evaluates the descriptive power of the visual synchronization task to measure mental fatigue.
\end{abstract}

\section{CCS Concepts}

- Applied computing $\rightarrow$ Consumer health

\section{Keywords}

Mental fatigue; brain function; motor control function, measurement.

\section{INTRODUCTION}

To increase our quality of life (QOL), we must control our mental fatigue along our mental works. We need a method to measure the mental fatigue objectively. This paper proposes a method to measure the performance decrease of our motor control function after a mental workload. Mental fatigue is not equal to a performance decrease of our motor control function. However, the performance of motor control function may take a role of objective measure of mental fatigue. This paper discusses the descriptive power of our visual synchronization task and its'

Permission to make digital or hard copies of all or part of this work for personal or classroom use is granted without fee provided that copies are not made or distributed for profit or commercial advantage and that copies bear this notice and the full citation on the first page. To copy otherwise, or republish, to post on servers or to redistribute to lists, requires prior specific permission and/or a fee.

ICBBS '17, June 22-24, 2017, Singapore, Singapore () 2017 Association for Computing Machinery.

ACM ISBN 978-1-4503-5222-2/17/06.. \$15.00

DOI: https://doi.org/10.1145/3121138.3121164 performance measure to observe a mental fatigue caused from a mental workload.

Many motor tasks measure the performances of motor control functions of a human. They are the Purdue pegboard task, a seal affixation task, a tray carrying task, and etc. [1,2,3] These tasks estimate the performance of a motor function of a human based on the results of the tasks. There is no observation of the process of the tasks.

There are also some synchronization tasks to measure a motor function of a human. One example is a synchronization of finger taps with periodically flashing visual stimuli and synchronization with an auditory metronome. In these tasks, the timing between the stimuli and the tapping is measured. There is no observation of the process of the tapping $[4,5,6,7,8,9,10]$.

Recently, many cheap and easy methods to measure the movements of a human body have been developed. For instance, some of these sensors include Kinect sensor, and Leap motion sensor [11, 12]. There are many applications that use those sensors for controlling computers. For instance, there are many video games that use those sensors for controlling an avatar in the games [13].

Using these new motion sensors, we can measure the motion of hands easily and precisely. The human hands are the parts of a body that can make the most complex movements. We have proposed a method that measures the precise movements of hands synchronizing the movements of hands on a display. The author calls this method visual synchronization task. The synchronization needs visual perception of the displayed hands' images and precise control of the arm muscles. The resulting measure is very sensitive. With this measure, we can evaluate the performance of the motor control function precisely.

For estimating a mental fatigue, we need a method to measure the performance of a mental work. Kraepelin test is one of a method to measure the performance of mental work [14]. However, Kraepelin test needs at least five minutes. In addition, the test itself is a heavy mental workload. Therefore, Kraepelin test is not fit to estimate a mental fatigue from a mental workload and to observe the recovery from the fatigue.

The visual synchronization task is very short to complete and very easy to do. Our visual synchronization task can measure the performance of motor control function in complex situation. Therefore, the visual synchronization task is able to measure the performance of mental work.

This paper discusses the performance of the visual synchronization task to measure the fatigue caused from a mental work. First, this paper discusses a task to synchronize hands' movements with visual presentation. Then, the authors propose the outline of the observation system to measure a visual 
synchronization of hands' motions, and our implementation of the observation system. Next, this paper discusses a trial to measure the fatigue caused by a mental workload. Then, the author proposes the experiments and their results. And last, this paper concludes this work.

\section{VISUAL SYNCHRONIZATION TASK $2.1 \quad$ Task}

Many motor tasks intend to measure the motor control function of a human. However, most of these tasks measure the results of the tasks. Some tasks measure the synchronization between a finger tap and stimuli. With human observations, it is difficult to measure the process of synchronizing movements. Now, we can use a Kinect sensor and a Leap Motion sensor [11, 12]. These sensors measure the three-dimensional movements of a human body. With these sensors, we can measure the precise movements of a human body.

We can synchronize our movements with each other. For instance, in playing a dance, dancers can synchronize their movements with each other. A synchronization of movement is more difficult work than a simple imitation of movements. To generate synchronized movements, we need to observe the motion to be synchronized. We need to generate the motion to be similar to the motion synchronized. We need to observe the generated motion synchronizing the original motion. We need to estimate the divergence between the original motion synchronized and the motion synchronizing the original motion. We need to control the speed of the motion synchronizing. These functions make the feedback loop. However, for compensating our brain's processing delay, we need to estimate the delay itself and to make feedforward. To make feedforward, we need to remember and to forecast the periodic motion.

This processing loop is shown in Figure 1. For estimating the total brain function, we need to include all the functions of a brain. The visual synchronization task includes vision and motor control functions. The vision includes not only the static sight, but also the dynamic sight.

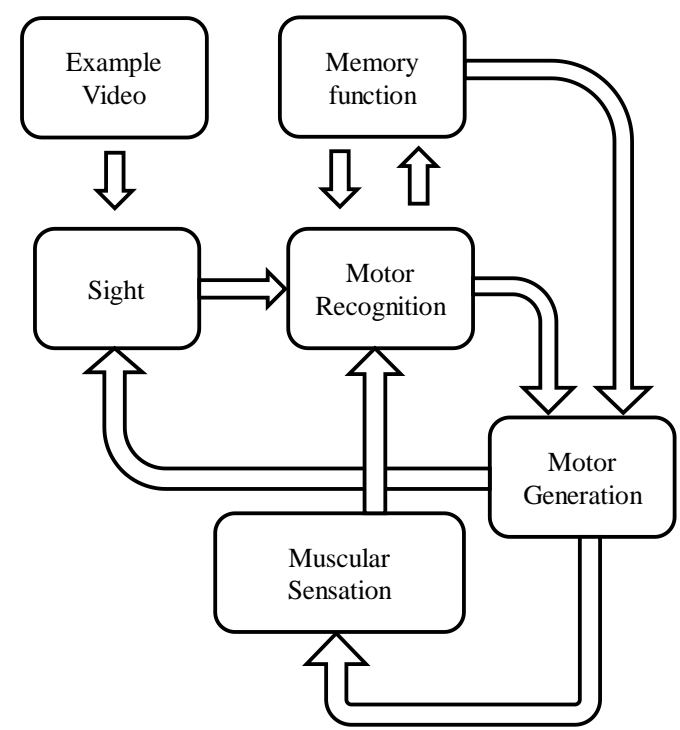

Figure 1. Relation among functions.

The visual synchronization is more difficult than audio synchronization. Therefore, we observe the wider brain functions with the visual synchronization tasks than the audio synchronization tasks.

Our proposed visual synchronization task is the synchronization between the position of stimuli as the displayed hands and the position of subject's hands. Our synchronization task is not the synchronization between the timing of the stimuli and the timing of action. The measurement of timing is only one scalar value. In our proposed synchronization task, the measurements of positions both of the stimuli and the hands are the sequence of a triple of the positions of the stimuli and the ones of both hands. In one cycle of movement, we have larger amount of measurements from the proposed visual synchronization task than the tapping test.

For the ease of processing measured data, our visual synchronization task uses the stimuli controlled as sine curve. This makes easy to estimate the performance of a subject.

\subsection{Mental Fatigue on Visual Synchronization Task}

Our visual synchronization task forces a subject to use many kinds of functions in a brain. Therefore, the performance of the visual synchronization task represents the over-all performance of a brain. A mental fatigue causes the decrease of the performance of a brain. Therefore, our visual synchronization task may measure the degree of mental fatigue.

There are Bourdon test and Kraepelin test that measures the performance of mental work. $[14,15,16]$ They need at least five minutes or more. They themselves are heavy mental workload. Therefore, it is difficult to complete many tests to measure the performance change before and after a metal workload.

Our visual synchronization task needs only 15 seconds to complete a measurement. We can repeat the visual synchronization task to measure the change of the performance of a brain before and after a mental workload. The shortness of our visual synchronization task enables to measure the sequence of the performance changes of a brain before and after a mental workload.

\subsection{Motor Control Function Measure}

We have large amount of measurements in a trial. We hope to show a simple scalar measure that represents the total brain activity. We define the motor control function measure using FFT (Fast Fourier Transform) results of the measured poses of both hands in each cycle. If a subject makes complete synchronization to the stimuli, the resulting measurements follow a complete sine curve. As a result, at every cycle in stimuli, the result of FFT has a zero value at the second term or higher terms. We define the measure as (1). This measure increases with the amount of the difference of measured data from ideal sine curve.

$$
N S M=\left(\sum_{x=2}^{t / 4} m_{x}\right) / m_{1}
$$

In (1), $t$ is the number of terms. $m_{x}$ is the absolute value of the $\mathrm{x}$ th term of the result of FFT. $m_{1}$ is the power of the lowest frequency. This represents a one cycle of a hand's rotation. If the rotation of a hand follows the stimuli images precisely, the $m_{1}$ carries all powers of the hand's rotation. Other terms carry no power. In the case, the measure in (1) is $0 . m_{0}$ is a value that 
represents the average of poses. This is not included in (1). As a result, this measure does not depend the absolute poses of hands.

We call this measure as Non-Smoothness-Measure (NSM). This measure may span from 0 to infinite. Our proposed system observes two hands. Therefore, at every cycle, we have two NSMs.

In other words, NSM represents the performance of a signal amplifier that input is visual stimuli and output is pose of a part of body.

\section{IMPLEMENTATION OF MEASUREMENT SYSTEM}

We can use many parts of our body for the output operand of our visual synchronization task. For instance, we can use the movement of a finger as a tapping test. However, to measure the performance of a brain, a movement must have some difficulty. A rotation of hand has a little difficulty. To make rotation, many muscles must be controlled coordinately. Many infants over five years old can rotate their hands. Under four years old, infants have difficulty to rotate their hands. Figure 2 shows the rotation of hands.

This paper selects rotation of hands as stimuli. Figure 2 shows the movements of hands. We can easily define the pose of hands using the normal of perms.

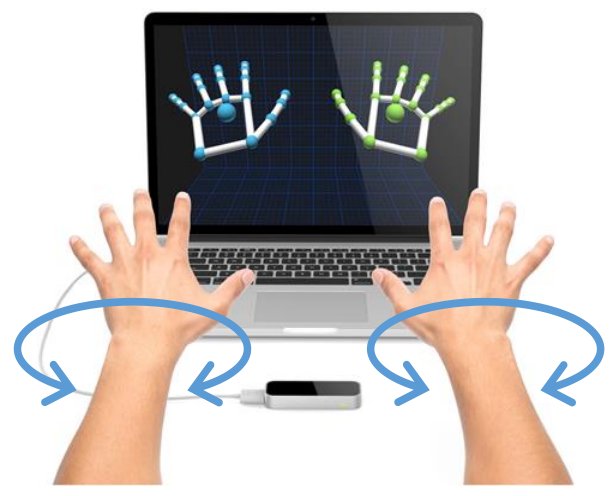

Figure 2. Movements of hands.
It is difficult to make rotation of hands to follow a sine curve. Therefore, our displayed hands' images are composed from video frames to follow a sine curve.

The author uses Python and Pyglet to implement the measuring system. [17, 18] Pyglet enables to control the real time presentation of stimuli images.

With leap motion sensor, our system measures the poses of both hands at each $1 / 100$ second with displaying the stimuli images. Therefore, our implementation of VST measures 100 tuples of the poses of both hands. Comparing with the tapping test, our implementation of VST gets 100 times of measurements. This leads to the precise estimation of a performance.

\section{MEASUREMENT OF MENTAL FATIGUE}

\subsection{Outline of Trial}

We can estimate the effect of some activities with comparing between the performance before an activity and one after the activity. We will measure mental fatigue, so we must select some activities that need mental works. Some tests measure the performance of mental works. For instance, Kraepelin test measure the performance of mental work. However, it need at least five minutes, and the test itself is a heavy mental workload. Therefore, Kraepelin test itself makes a subject to be tired. Our visual synchronization task needs only 15 seconds to perform. There is a little influence about a performance of motor control function.

Our trial has three parts. One part has five measurements of the visual synchronization task (VST). Each VST needs only 15 seconds. There is four minutes 45 seconds rest between adjacent VSTs. Second part is a game playing. The author selects a fighting video game for mental workload. The last part is 13 measurements of VSTs. Figure 3 shows the structure of a trial to measure the fatigue of mental work. This trial needs about two hours to complete.

Over all trial need about two hours to be completed. We hope to measure longer period. However, this two hours' trial is difficult to complete.

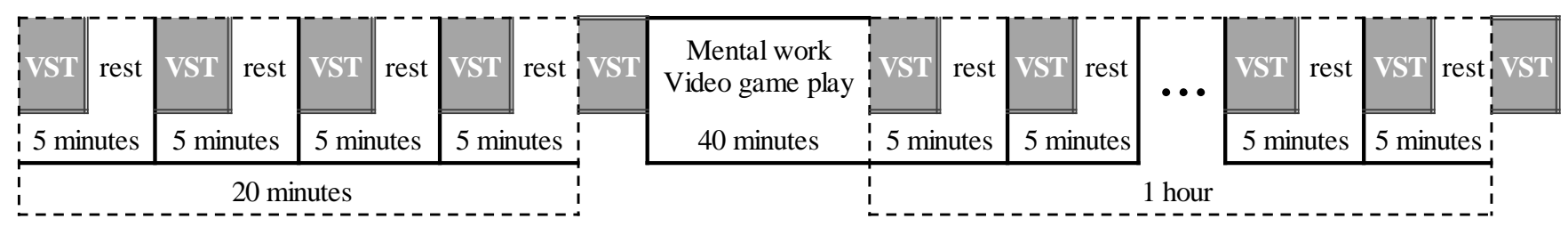

Figure 3. Structure of a trial.

\subsection{Workload}

The author uses an action video game for a mental workload. The author selects Japanese version of "Super Smash Bros." The game is hilarious fighting game. Therefore, a subject concentrate to play this game. A subject enjoys playing this game. Therefore, a subject can play continuously for 40 minutes without large difficulty.

Kraepelin test is used widely for mental workload and for performance measuring method. However, as a mental workload, Kraepelin test is boring. The test fits for measuring the concentration in boring work. However, a subject has difficulty to keep concentration.

The selected video game fit for a mental workload. We can easily keep concentration in playing the game.

\subsection{Performance Measure}

At each VST, we have 15 pairs of NSMs defined as (1). However, we hope to have a single value that represents the performance of the VST. There are 30 NSMs. However, we hope to define a 
scalar measure that represents the performance of brain activity of a subject at a VST from the 30 NSMs.

Our previous experiments show that the difference between a left hand and a right hand is small. However, we treat both hands. There is 15 NSMs at a hand. We can use the mean to define the scalar measure of a brain activity. However, it is difficult to keep the hands movements in 15 seconds. There is a difference among 30 NSMs. We can use the mean of all 30 NSMs. However, there are some error measurements. Our experiments show that the first cycle shows big NSMs, because a subject try to synchronize his hands' movements to the displayed hands' movements. There is a small NSM caused from accidentally matching between the displayed movement and the subject's movement.

We define the NSM of a trial as (1). We call the NSM of a VST as BVNSM in the following.

$$
\begin{aligned}
& \mathrm{BVNSM}= \\
& \min _{i=1,13, h=\{l, r\}} \operatorname{average}\left(N S M_{h, i}, N S M_{h, i+1}, N S M_{h, i+2}\right)
\end{aligned}
$$

In (2), $N S M_{h, i}$ is a $N S M$ of a hand $h$ at a cycle $i$. The BVNSM shows the best performance of a subject in a trial. We use this BVNSM for evaluating the performance of a subject in a trial. In simply, the BVNSM is the best performance of a subject in a trial. There are persons that can only keep their hands movements properly in a very short period. Those persons may have some kinds of problems about their brain functions. However, we only treat a brain performance in a short period. The BVNSM defined as (2) represents the best performance of a VST.

From other viewpoint, we need the measure that represents the average performance of a VST. In a VST, we have 30 NSMs that include some error measurements. We must remove these error measurements. Therefore, we select the average of $80 \%$ middle range values in the set of NSMs. The formal definition is (3).

$$
\operatorname{AVNSM}=\underset{i=4,12}{\text { average }}(\operatorname{sort}(N S M s))
$$

In (3), NSMs is the set of right hand's NSMs in a VST. The average processes only from fourth largest value to 12-th largest value. This AVNSM may represents the average performance of a VST.

\section{EXPERIMENTS AND RESULTS}

\subsection{Subjects and Trials}

Experiments include six subjects. They made 33 trials. All subjects are male students. Their ages are from 23 to 25 years old.

\subsection{Experimental Results}

\subsubsection{AVNSMs}

Figure 4 shows the all AVNSMs in 33 trials. In Figure 4, a shaded box is the position of the mental workload. The $\mathrm{x}$-axis is the sequence number of VSTs. The y-axis is AVNSMs. Before and after the workload, there is no apparent difference in the distribution. Table 1 shows the total of measurements of AVNSMs before workload. Table 2 shows the total measurements of AVNSMs after workload. Comparing before and after the workload, the average of AVNSMs before the workload is less than the one after the workload. Figure 5 show the change of average AVNSMs. In Figure 5, a shaded box is the position of the 40 minutes mental workload. After the mental workload, there is a little increase of AVNSMs. However, the difference is not apparent. Table 3 shows the t-test's result of average AVNSMs between before and after the workload. The t-test confirms the difference between AVNSMs before and after the workload with three percent critical region. The both side probability is 0.0061 .

Table 1. Total of AVNSM before Workload.

\begin{tabular}{|c|c|c|}
\hline \#VST & \multicolumn{1}{c|}{ Mean } & \multicolumn{1}{c|}{ STD } \\
\hline 1 & 0.318257 & 0.068197 \\
\hline 2 & 0.31948 & 0.105438 \\
\hline 3 & 0.328394 & 0.097062 \\
\hline 4 & 0.329042 & 0.077119 \\
\hline 5 & 0.353634 & 0.130244 \\
\hline Total & 0.329762 & 0.095612 \\
\hline
\end{tabular}

Table 2. Total of AVNSM after Workload

\begin{tabular}{|c|c|c|}
\hline \#VST & \multicolumn{1}{c|}{ Mean } & \multicolumn{1}{c|}{ STD } \\
\hline 1 & 0.327914 & 0.073945 \\
\hline 2 & 0.351448 & 0.178446 \\
\hline 3 & 0.339894 & 0.092166 \\
\hline 4 & 0.385488 & 0.183024 \\
\hline 5 & 0.371683 & 0.160763 \\
\hline 6 & 0.361417 & 0.17388 \\
\hline 7 & 0.335335 & 0.0851 \\
\hline 8 & 0.396002 & 0.281234 \\
\hline 9 & 0.350735 & 0.125879 \\
\hline 10 & 0.357451 & 0.124321 \\
\hline 11 & 0.412456 & 0.279172 \\
\hline 12 & 0.339067 & 0.091774 \\
\hline 13 & 0.357681 & 0.095419 \\
\hline Total & 0.360505 & 0.149625 \\
\hline
\end{tabular}

Table 3. Result of t-test between AVNSMs before and after a workload

\begin{tabular}{|l|r|r|}
\hline \multicolumn{1}{|c|}{ Feature } & \multicolumn{1}{c|}{ Before } & \multicolumn{1}{c|}{ After } \\
\hline Average & 0.3298 & 0.3605 \\
\hline Distribution & 0.0002026 & 0.0006236 \\
\hline
\end{tabular}




\begin{tabular}{|l|r|r|}
\hline \# of samples & 5 & 13 \\
\hline Freedom & 13 & \\
\hline $\mathrm{t}$ & -3.2682 & \\
\hline $\mathrm{P}(\mathrm{T}<\mathrm{t})$ both side & 0.006110 & \\
\hline
\end{tabular}

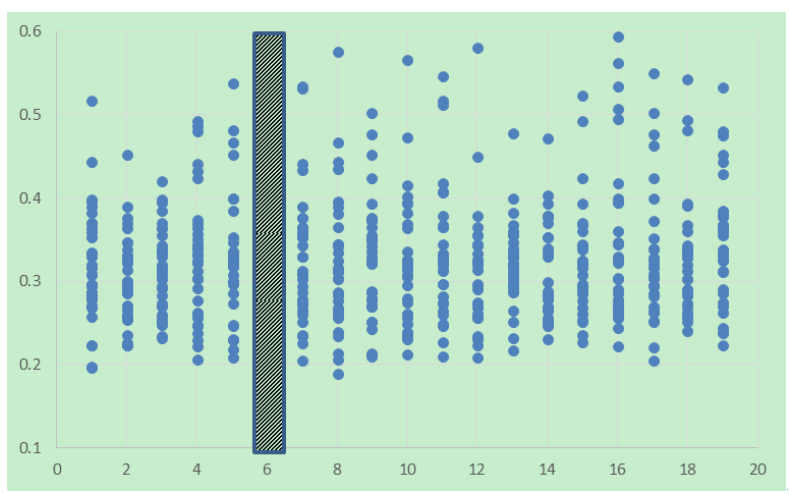

Figure 4. Distribution of AVNSMs.

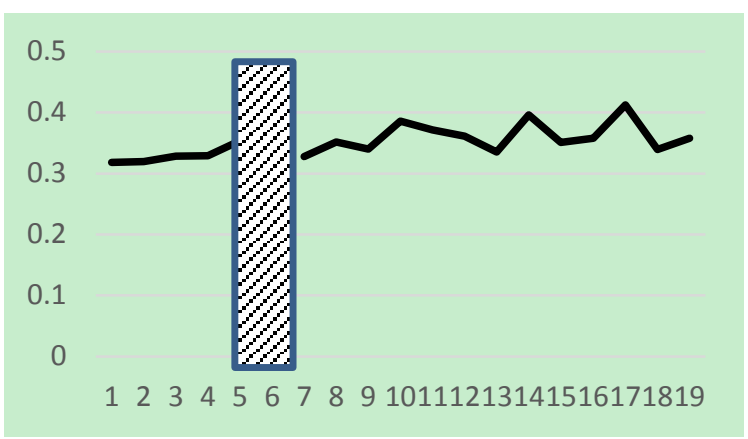

Figure 5. Average AVNSMs in Total Trials.

The average AVNSMs just after the workload period is smaller than one just before the workload. After the workload period that includes 13 VSTs, the average AVNSMs increase a little. If the performance of a brain reflect only the mental fatigue, the AVNSM just after the workload period must largest of all. However, we can see a little decrease of AVNSMs after the workload period. Our mental workload is very exciting fighting video game. Therefore, a subject is excited in playing the game. In excited state, the performance of a brain is boosted. Our trial ends only one hour after the game playing. In the whole period, a subject's excitements is cooling down. Therefore, our trials cannot measure the process recovering from the mental fatigue.

NSM is the measure that decreases with better performance. Therefore, AVNSM also decreases with better performance. The increase of AVNSM shows the fatigue from the workload. The difference between before and after the workload is confirmed with three percent critical region. This concludes that AVNSM can detect a mental fatigue.

Table 4 shows the result of t-test between the sets of AVNSMs before and after the workload. The t-test's result confirms that the apparent difference between the set of AVNSMs before and after the workload. The both side probability is 0.0069 . This leads that the AVNSM can observe the mental fatigue.

Table 4. Result of t-test between the sets of AVNSMs before and after a workload

\begin{tabular}{|l|r|r|}
\hline \multicolumn{1}{|c|}{ Feature } & \multicolumn{1}{c|}{ Before } & \multicolumn{1}{c|}{ After } \\
\hline Average & 0.3307 & 0.3610 \\
\hline Distribution & 0.009764 & 0.02755 \\
\hline$\#$ of samples & 165 & 417 \\
\hline Freedom & 493 & \\
\hline $\mathrm{t}$ & -2.712 & \\
\hline $\mathrm{P}(\mathrm{T}<=\mathrm{t})$ both side & 0.006922 & \\
\hline
\end{tabular}

\subsubsection{BVNSMs}

Figure 6 shows the distribution of all BVNSMs in 33 trials. In Figure 6, the $\mathrm{x}$-axis is the sequence number of VSTs. The y-axis is BVNSMs. A shaded box is the position of the 40 minutes mental workload. Before and after the workload, there is no apparent difference in the distribution. Table 5 shows the total of measurements of BVNSMs before workload. Table 6 shows the total measurements of BVNSMs after workload. Figure 7 shows the change of average BVSMs. Comparing before and after the workload, the average of BVNSMs before the workload is less than the one after the workload. Table 7 shows the result of the ttest. The difference between before and after the workload is confirmed with five percent critical region. This concludes that BVNSM can detect a mental fatigue. However, BVNSM is weaker than AVNSM in total for observing a mental fatigue.

Table 5. Total of BVNSM before Workload

\begin{tabular}{|c|c|c|}
\hline \#VST & Mean & STD \\
\hline 1 & 0.21680 & 0.02417 \\
\hline 2 & 0.22284 & 0.03230 \\
\hline 3 & 0.22922 & 0.02903 \\
\hline 4 & 0.23518 & 0.03574 \\
\hline 5 & 0.22224 & 0.02907 \\
\hline Total & 0.22525 & 0.03006 \\
\hline
\end{tabular}

Table 6. Total of BVNSM after Workload

\begin{tabular}{|c|l|l|}
\hline \#VST & \multicolumn{1}{l|}{ Mean } & \multicolumn{1}{l|}{ STD } \\
\hline 1 & 0.23041 & 0.03651 \\
\hline 2 & 0.22378 & 0.03317 \\
\hline 3 & 0.23297 & 0.03229 \\
\hline 4 & 0.22991 & 0.03500 \\
\hline
\end{tabular}




\begin{tabular}{|c|r|r|}
\hline 5 & 0.23778 & 0.03363 \\
\hline 6 & 0.23729 & 0.04130 \\
\hline 7 & 0.23599 & 0.02874 \\
\hline 8 & 0.23196 & 0.02927 \\
\hline 9 & 0.23666 & 0.03194 \\
\hline 10 & 0.23559 & 0.02709 \\
\hline 11 & 0.23880 & 0.03175 \\
\hline 12 & 0.24354 & 0.03226 \\
\hline 13 & 0.23549 & 0.03177 \\
\hline Total & 0.23459 & 0.03267 \\
\hline
\end{tabular}

Table 7. Result of t-test between BVNSMs before and after a workload

\begin{tabular}{|l|r|r|}
\hline \multicolumn{1}{|c|}{ Feature } & \multicolumn{1}{c|}{ Before } & \multicolumn{1}{c|}{ After } \\
\hline Average & 0.2253 & 0.2346 \\
\hline Distribution & $5.019 \mathrm{E}-05$ & $2.409 \mathrm{E}-05$ \\
\hline \# of samples & 5 & 13 \\
\hline Freedom & 6 & \\
\hline $\mathrm{t}$ & -2.718 & \\
\hline $\mathrm{P}(\mathrm{T}<=\mathrm{t})$ both side & 0.03475 & \\
\hline
\end{tabular}

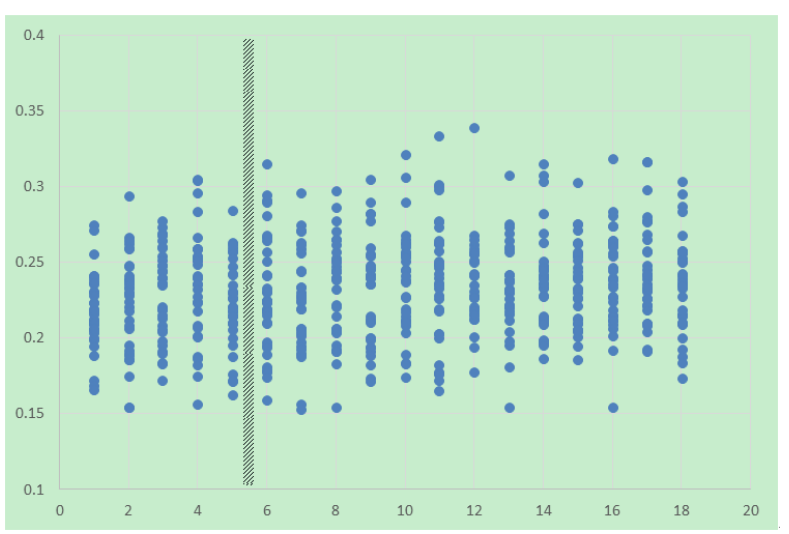

Figure 6. Distribution of BVNSM.

It is checked the sets of all BVMSNs before and after a workload with the t-test. Table 8 shows the result of the t-test. The probability of both-side is 0.000817 . This is small enough to confirm the precision of the proposed VST to measure the performance of our brain function. With AVNSMs, table 4 shows that the probability is 0.0069 . The BVNSMs' difference between the performances before and after the workload is more apparent than one of AVNSMs. BVNSM is better to measure the fatigue with mental workload than AVNSM.

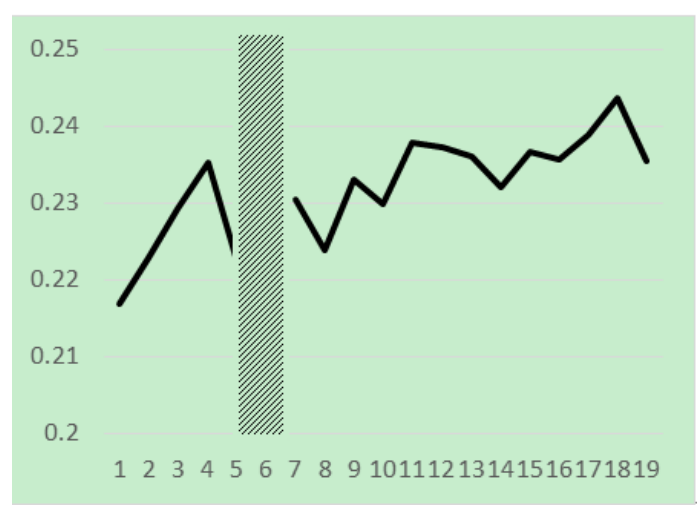

Figure 7. Average BVNSMs in Total Trials.

Table 8. Result of t-test between the sets of BVNSMs before and after a workload

\begin{tabular}{|l|r|r|}
\hline \multicolumn{1}{|c|}{ Feature } & \multicolumn{1}{c|}{ Before } & \multicolumn{1}{c|}{ After } \\
\hline Average & 0.2248 & 0.2346 \\
\hline Distribution & 0.0009745 & 0.001106 \\
\hline$\#$ of samples & 169 & 421 \\
\hline Freedom & 329 & \\
\hline $\mathrm{t}$ & -3.378 & \\
\hline $\mathrm{P}(\mathrm{T}<=\mathrm{t})$ both side & 0.0008173 & \\
\hline
\end{tabular}

There are personal difference among subjects. However, the distributions of measured BVNSMs represents the difference between the performances before and after the metal workload.

\section{CONCLUSION}

Our visual synchronization task has enough descriptive power to measure the performance decrease caused from a mental workload. Our visual synchronization task is a light workload. With our visual synchronization task, we can measure the process of fatigue recovery. Because, our visual synchronization task needs only 15 seconds to complete. Our visual synchronization task is lighter mental workload than classical mental performance measuring methods.

The t-tests confirms that the AVNSM and BVNSM have enough descriptive power to measure the mental fatigue. However, which one is better measure to evaluate the mental fatigue is not known.

In our experiments, we can follow the change of the performance of a brain after a mental workload. However, this paper cannot analyze precise changes of the performance. This is our next step.

\section{ACKNOWLEDGMENTS}

The author thanks Mr. H. Hotta for his works to make experiments. This work is supported with JSPS16K01057 and JSPS16K04818.

\section{REFERENCES}

[1] Lafayette Instrument, 2015. Purdue Pegboard Test, http://www.lafayetteevaluation.com/product_detail.asp?ItemI $\mathrm{D}=159$, retrieved 2015/07/07 
[2] Kokubun, M. 1999. Are children with down syndrome less careful in performing a tray-carrying task than children with other types of mental retardation? Perceptual and Motor Skills. 88:3c, 1173-1176.

[3] Vercruysse, S., et al. 2012. Freezing in Parkinson's disease: a spatiotemporal motor disorder beyond gait. Movement Disorders. 27(2), 254-263.

[4] Hirata, S., Kitajima, Y., Hosobuchi, T., and Kokubun, M. 2008. The speed and accuracy of fine motor actions in children with intellectual disabilities. Bulletin of Tokyo Gakugei University, 59, 263-267.

[5] Sugano, Y., Keetels, M., and Vroomen, J. 2012. The build-up and transfer of sensorimotor temporal recalibration measured via a synchronization task. Front Psychol. 3, 246, DOI: 10.3389/fpsyg.2012.00246.

[6] Rubia, K., Smith, A. B., Brammer, M. J., Toone, B., and Taylor, E. 2005. Abnormal brain activation during inhibition and error detection in medication-naïve adolescents with ADHD. Am Psychiatric Assocvolume. 162(6), 1067-1075.

[7] Pyglet, 2015. pyglet, http://www.pyglet.org/, retrieved 2015/03/01.

[8] Kuratsune H. 2009. Changes in autonomic nerve function in the mental fatigue state caused by long-term computerized Kraepelin test workload. Symposium Molecular/neural mechanisms of fatigue and fatigue sensation, The $36^{\text {th }}$ Congress of the International Union of Physiological Sciences (Kyoto).

[9] Koreaki, S., Aya, K., and Noriaki, S. 2009. The effectiveness of the Uchida-Kraepelin test for psychological stress: an analysis of plasma and salivary stress substances.

BioPsychoSocial Medicine. 3(5), http://bpsmedicine.biomedcentral.com/articles/10.1186/1751 -0759- 3-5, retrieved at 2017/01/24.

[10] Kurahashi, S. 1957. Development of the Uchida-Kraepelin psychodiagnostic test in Japan. Psychologia (Kyoto). 1957(1), 104-109.

[11] Smith, M. A., Brandt, J., Shadmehr, R. 2000. Motor disorder in Huntington's disease begins as a dysfunction in error feedback control. Nature. 403, 544-549.

[12] Microsoft. 2015. Kinect for Windows, https://www.microsoft.com/en-us/kinectforwindows/, retrieved 2015/0707.

[13] Leap Motion. 2015. Leap Motion SDK, https://developer.leapmotion.com/, retrieved at 2015/03/17.

[14] Lee, J., Chai, J., and Reitsma, P. S. A. 2002. Interactive control of avatars animated with human motion data. ACM Transactions on Graphics (TOG). ACM. 491-500.

[15] Hove, M. J., Iversen, J. R., Zhang, A. and Repp, B. H. 2013. Synchronization with competing visual and auditory rhythms: bouncing ball meets metronome. Psychological Research. 77, 388-398.

[16] Krause, V., Pollok, B., and Schnitzler, A. 2010. Perception in action: The impact of sensory information on sensorimotor synchronization in musicians and non-musicians. Acta Psychologica. 133(1), 28-37.

[17] Microsoftstore. 2015. Kinect, http://www.microsoftstore.com/store/ msusa/en_US/list/Kinect/categoryID.64752700, retrieved 2015/07/08.

[18] Python. 2015. Python, https://www.python.org/, retrieved 2015/03/17. 